\title{
Necessary cautions when considering digoxin in heart failure
}

\author{
George A. Heckman, Robert S. McKelvie
}

$\infty$

See related article page $64 \mathrm{I}$

$\mathrm{D}$ igitalis is an old remedy. The first systematic study showing the benefits of digitalis in cases of dropsy was reported by the English physician and botanist William Withering in $1785 .{ }^{1}$ Subsequent widespread use of digitalis led to a greater understanding of its mechanisms of action: but until recently, the evidence on its clinical efficacy in heart failure was derived from small, often uncontrolled trials. The only adequately powered study to date to assess the impact of digoxin on survival in heart failure remains the Digitalis Investigation Group (DIG) trial. ${ }^{2}$ Multiple post-hoc analyses of the DIG trial have been undertaken to determine the efficacy of digoxin in a variety of subpopulations, the results of which continue to fuel debate over its role in the management of heart failure.

The DIG study consisted of 2 randomized, placebocontrolled trials. The main trial assessed the effect of digoxin on mortality and hospital admission in 6800 patients with heart failure, a left-ventricular ejection fraction (LVEF) less than $45 \%$, and predominantly New York Heart Association (NYHA) class II-III symptoms. After a mean follow-up of 37 months, the difference in death rate was found to be nonsignificant, although patients who received digoxin were less likely to be admitted to hospital for worsening heart failure (risk ratio $0.72,95 \%$ confidence interval o.66-0.79). The ancillary DIG trial assessed another 988 patients with heart failure, but with preserved systolic function (LVEF > 45\%) and symptoms that fell primarily into NYHA classes I or II. ${ }^{3} \mathrm{Al}-$ though an interim analysis at 2 years suggested that patients randomly assigned to the digoxin treatment group had lower risks of death and hospital admission, the final results of the ancillary trial (after a mean follow-up of 37 months) demonstrated no overall effect. This result was consistent with a statistical phenomenon akin to regression to the mean, whereby early differences eventually lose their statistical significance with longer follow-up., ${ }^{3,4}$

The results of several post-hoc subgroup analyses of the DIG trials have been inconsistent. A pooled analysis ${ }^{5}$ of the main and ancillary trials suggested that the effects of digoxin were independent of age. An analysis of the main trial ${ }^{6}$ suggested that digoxin therapy in women but not men was associated with a higher death rate, although a subsequent reanalysis ${ }^{7}$ found that this mortality excess was associated with digoxin concentrations in serum of $1.2 \mathrm{ng} / \mathrm{mL}$ or greater (lower concentrations had a neutral effect on mortality). Another analysis of the main trial found a statistically nonsignificant trend toward greater mortality among men whose serum digoxin concentrations were $\mathrm{I} .2 \mathrm{ng} / \mathrm{mL}$ or more. ${ }^{8}$
The most recent re-analysis of the DIG trials by Ahmed and colleagues ${ }^{9}$ explores the effects of digoxin in participant subgroups based on sex, age $\geq 70$, ethnic origin, NYHA class, LVEF $>45 \%$, etiology of heart failure, the presence of chronic renal disease or diabetes, concurrent pharmacotherapy for heart failure and serum digoxin concentrations $\geq 1.0 \mathrm{ng} / \mathrm{mL}$. The re-analysis was restricted to 1687 patients treated with digoxin, for whom serum digoxin concentrations were available I month after randomization, and 386r placebo-treated participants who survived to that time. The effect of serum digoxin concentrations was assessed by matching each participant in the digoxin group with one from the placebo group and comparing outcomes within each pair. Pairing was based on propensity scores that reflected the probability of a participant developing a high or low serum digoxin concentration, given baseline characteristics. The analysis suggested that in all subgroups, lower serum digoxin concentrations were associated with fewer deaths or hospital admissions for heart failure, compared with placebo. The authors concluded that digoxin was beneficial in heart failure where systolic function was preserved, and furthermore suggested that in all heart failure patients who remain symptomatic despite optimal therapy with angiotensin-converting enzyme inhibitors and $\beta$-blockers, digoxin should be considered ahead of angiotensin receptor blockers, aldosterone antagonists or other proven therapies. $^{9}$

\section{Findings from subgroup analyses have been inconsis-} tent, and need verification.

These conclusions are not justified given the quality of the evidence available, as ascertained via published criteria for evaluating subgroup analyses..$^{10}$ First, positive results from multiple post-hoc subgroup analyses may arise purely by chance. Second, the claim that digoxin reduces mortality in heart failure with preserved systolic function was based on the lack of a statistically significant interaction between leftventricular function and serum digoxin concentrations in the analysis, although the actual confidence interval for the hazard ratio for death in the group with low serum digoxin concentrations and preserved systolic function was large and included unity. ${ }^{9}$ Third, the findings from other subgroup 
analyses, particularly those pertaining to interactions between sex and serum digoxin concentration, are not entirely consistent, which further weakens the conclusions by Ahmed's group. Fourth, the subgroup analysis by Ahmed and colleagues included only $7 \mathrm{I} \%$ of the original DIG trial cohort, and the propensity score analysis, only $43 \%$ of the cohort. The questionable generalizability of these results is further compounded when the characteristics of the DIG trial cohort are considered. Typical populations of people with heart failure are aged well into their late 7os and early 8os, have substantial comorbidity and include a greater proportion of women and nonwhite people than those in the DIG trial, who were primarily white, male and an average of 64 years of age. ${ }^{2,11}$ Older patients who undergo heart failure are at increased risk for side effects from digoxin, which are often nonspecific and can occur at serum digoxin concentrations generally considered therapeutic. ${ }^{12,13}$ Finally, there are no head-to-head trials on which to base the contention that digoxin should be used ahead of other heart-failure therapies that have been supported by dedicated randomized controlled trials large enough to be adequately powered. ${ }^{14}$

The 2006 Canadian Cardiovascular Society guidelines on heart failure recommend the use of digoxin in patients with heart failure due to left-ventricular systolic dysfunction who remain symptomatic despite optimal therapy, or in those with heart failure accompanied by chronic atrial fibrillation and poorly controlled ventricular rate despite $\beta$-blocker therapy or who cannot tolerate $\beta$-blockers. ${ }^{14}$ The guidelines suggest that digoxin may be considered to minimize symptoms in heart failure with preserved systolic function, with the caveat that the efficacy of this intervention is less well established than that of other therapies, including angiotensin-converting enzyme inhibitors, $\beta$-blockers and angiotensin receptor blockers. Evidence from the multiple subgroup analyses of the DIG trial is insufficient to warrant any changes to these recommendations.

The problem of heart failure with preserved systolic function is an important one. The findings from subgroup analyses of the DIG trial must be considered preliminary, and should be verified in an adequately powered, randomized controlled trial that includes a representative sample of older patients and examines low serum digoxin concentrations. The fact that digoxin is a nonpatentable remedy should not be a deterrent to the conduct of such a trial, which would require funding by public agencies. The inability of some patients with heart failure to afford effective therapies such as angiotensin-converting enzyme inhibitors and $\beta$-blockers is deplorable, although the ultimate remedy to that problem lies in more equitable distribution of health care resources (both nationally and abroad) rather than recommendations of therapies whose evidence is less substantial.

\section{This article has been peer reviewed.}

George Heckman is with the Divisions of Geriatric Medicine and Cardiology, and Robert McKelvie is with the Division of Cardiology, Department of Medicine, McMaster University, Hamilton, Ont.

Competing interests: Both authors were on the primary panel that critically reviewed new clinical trial evidence and meta-analyses on which the 2006 Canadian Cardiovascular Society consensus guidelines on heart failure were based. Otherwise, they have no relevant conflicts of interest to declare.

Contributors: Both authors contributed to the content of the commentary and gave final approval of the version to be published.

\section{REFERENCES}

I. Somberg J, Greefield B, Tepper D. Digitalis: historical development in clinical medicine. J Clin Pharmacol I985;25:484-9.

2. Digitalis Investigation Group. The effect of digoxin on mortality and morbidity in patients with heart failure. N Engl J Med I997;336:525-33.

3. Ahmed A, Rich MW, Fleg JL, et al. Effects of digoxin on morbidity and mortality in diastolic heart failure: the ancillary Digitalis Investigation Group trial. Circulation 2006;1I4:397-403.

4. $\quad$ Pocock S, White I. Trials stopped early: Too good to be true? Lancet 1999;353:943-4.

5. Rich MW, McSherry F, Williford WO, et al. Effect of age on mortality, hospitalizations and response to digoxin in patients with heart failure: the DIG study. J Am Coll Cardiol 2001; 38:806-13.

6. Rathore SS, Wang Y, Krumholz HM. Sex-based differences in the effect of digoxin for the treatment of heart failure. N EngIJ Med 2002;347:I403-II.

7. Adams KF, Patterson JH, Gattis WA, et al. Relationship of serum digoxin concentration to mortality and morbidity in women in the Digitalis Investigation Group trial. J Am Coll Cardiol 2005;46:497-504.

8. Rathore SS, Curtis JP, Wang Y, et al. Association of serum digoxin concentration and outcomes in patients with heart failure. JAMA 2003;289:87I-8.

9. Ahmed A, Rich MW, Lover TE, et al. Digoxin and reduction in mortality and hospitalization in heart failure: a comprehensive post hoc analysis of the DIG trial. Eur Heart J 2006;27:178-86.

Io. Oxman A, Guyatt G. Summarizing the evidence: when to believe a subgroup analysis. Chapter $2 \mathrm{E}$ in: Guyatt $\mathrm{G}$, Rennie D, editors. Users' guides to the medical literature: a manual for evidence-based practice. Chicago: AMA Press; 2002. p. 553-65.

II. Heckman GA, McKelvie RS, Turpie ID. Heart failure in the frail elderly. Chapter 7 in: Turpie ID, Heckman GA, editors. Aging issues in cardiology. Boston: Kluver Academic Publishers; 2004. p. 139-62.

I2. Hanratty CG, McGlinchey P, Johnston GD, et al. Differential pharmacokinetics of digoxin in elderly patients. Drugs Aging 2000;17:353-62

13. Miura T, Kojima R, Sugiura Y, et al. Effect of aging on the incidence of digoxin toxicity. Ann Pharmacother 2000;34:427-32.

I4. Arnold JMO, Liu P, Demers C, et al; Canadian Cardiovascular Society. Canadian Cardiovascular Society consensus conference recommendations on heart failure 2006: diagnosis and management. Can J Cardiol 2006;22:23-45.

Correspondence to: Dr. George A. Heckman, Divisions of Geriatric Medicine and Cardiology, Department of Medicine, McMaster University, Hamilton Health Services, General Division, 4 East 419, 237 Barton St. East, Hamilton ON L8L 2X2; 905 527-4322 x4476o; heckO@sympatico.ca

\section{TRADITION}

Since I9II, CMAJ has been committed to advancing the science and art of medicine and promoting public health. 